\title{
Utility of driving pressure and mechanical power to guide protective ventilator settings in two cohorts of adult and pediatric patients with acute respiratory distress syndrome: A computational investigation
}

Sina Saffaran, $\mathrm{MSc}^{1}$; Anup Das, $\mathrm{PhD}^{1}$; John G. Laffey, $\mathrm{MD}^{2}$, Jonathan G. Hardman, $\mathrm{PhD}^{3}$; Nadir Yehya, $\mathrm{MD}^{4 *}$; and Declan G. Bates, $\mathrm{PhD}^{1 *}$

1 - School of Engineering, University of Warwick, Coventry CV4 7AL, UK.

2 - Anaesthesia and Intensive Care Medicine, School of Medicine, NUI Galway, Ireland.

3 - Anaesthesia \& Critical Care, Division of Clinical Neuroscience, School of Medicine, University of Nottingham, Nottingham NG7 2UH, UK.

4 - Department of Anaesthesiology and Critical Care Medicine, Children's Hospital of Philadelphia, University of Pennsylvania, Philadelphia, PA, USA.

* Corresponding Authors: yehyan@email.chop.edu (215-590-5907); D.Bates@ warwick.ac.uk (44-7876-876-952)

Clinical data were collected at the Children's Hospital of Philadelphia (CHOP) and the study was performed at The University of Warwick.

Funding: DGB and JGH acknowledge funding from the UK Engineering and Physical Sciences Research Council (Grant No. EP/P023444/1). NY acknowledges funding from the NIH (Grant No. NIH K23 HL-136688). 
Keywords: Adult ARDS; Pediatric ARDS; Mechanical ventilation; Ventilator-induced lung injury; Protective ventilation; Computer simulation.

Conflict of Interest: The authors declare that they have no conflicts of interest. 


\begin{abstract}
Objectives: Mechanical power (MP) and driving pressure $(\Delta \mathrm{P})$ have been proposed as indicators, and possibly drivers, of ventilator-induced lung injury. We tested the utility of these different measures as targets to derive maximally protective ventilator settings.
\end{abstract}

Design: A high-fidelity computational simulator was matched to individual patient data and used to identify strategies that minimize $\triangle \mathrm{P}, \mathrm{MP}$ and a modified version of MP (MMP) that removes the direct linear, positive dependence between MP and PEEP.

Setting: Interdisciplinary Collaboration in Systems Medicine Research Network.

Subjects: Data were collected from a prospective observational cohort of pediatric ARDS from the Children's Hospital of Philadelphia $(\mathrm{N}=77)$ and from the low tidal volume $\left(\mathrm{V}_{\mathrm{T}}\right)$ arm of the ARDSNetwork tidal volume trial $(\mathrm{N}=100)$.

Interventions: Global optimization algorithms evaluated more than 26.7 million changes to ventilator settings (approximately 150,000 per patient) to identify strategies that minimize $\Delta \mathrm{P}$, MP or MMP.

Measurements and Main Results: Large average reductions in $\Delta \mathrm{P}$ (23\%-Pediatric, $23 \%$-Adult), MP (44\%-Pediatric, 66\%-Adult) and MMP (61\%-Pediatric, 67\%-Adult) were achievable in both cohorts when oxygenation and ventilation were allowed to vary within pre-specified ranges. Reductions in $\triangle \mathrm{P}(12 \%$-Pediatric, $2 \%$-Adult), MP (24\%-Pediatric, 46\%-Adult) and MMP (44\%- 
Pediatric, 46\%-Adult) were achievable even when no deterioration in gas exchange was allowed. Minimization of MP and MMP was achieved by increasing $\mathrm{V}_{\mathrm{T}}$ and decreasing respiratory rate (RR). In the pediatric cohort, minimum $\Delta \mathrm{P}$ was achieved by reducing $\mathrm{V}_{\mathrm{T}}$ and increasing $\mathrm{RR}$ and PEEP. The ARDSNetwork dataset had limited scope for further reducing $\mathrm{V}_{\mathrm{T}}$, but $\Delta \mathrm{P}$ was still significantly reduced by increasing PEEP.

Conclusions: Our analysis identified different strategies that minimized $\Delta \mathrm{P}$ or MP consistently across pediatric and adult datasets. Minimizing standard and alternative formulations of MP led to significant increases in $\mathrm{V}_{\text {T. }}$. Targeting $\Delta \mathrm{P}$ for minimisation resulted in ventilator settings that also reduced MP and MMP, but not vice versa. 


\section{INTRODUCTION}

Mechanical power (MP) (1-3) and driving pressure ( $\Delta \mathrm{P})(4)$ have recently been proposed as measures, and potentially drivers, of ventilator-induced lung injury (VILI) in acute respiratory distress syndrome (ARDS). MP is defined as (1)):

$$
M P=0.098 \times R R \times\left\{V_{T}^{2} \times\left[0.5 \times E L_{r s}+R R \times \frac{(1+I: E)}{60 \times I: E} \times R_{a w}\right]+V_{T} \times P E E P\right\}
$$

where $\mathrm{EL}_{\mathrm{rs}}$ is the elastance of the respiratory system, I:E is the inspiratory-to-expiratory time ratio, and $\mathrm{R}_{\mathrm{aw}}$ is the airway resistance. $\Delta \mathrm{P}$ is defined as the difference between plateau pressure $\left(\mathrm{P}_{\text {plat }}\right)$ and PEEP, and reflects the tidal volume $\left(\mathrm{V}_{\mathrm{T}}\right)$ normalized to respiratory system compliance.

Arguments for the importance of MP focus on the injurious biophysical role of energy (stress X strain) and dynamics (rates of airway pressure change and cycling frequency) during mechanical ventilation (2), whereas arguments for the centrality of $\Delta \mathrm{P}$ are supported by statistical and computational analyses of trial data that show strong correlations between $\Delta \mathrm{P}$ and mortality $(4,5)$. However, the rationale for both MP and $\Delta \mathrm{P}$ rely on re-analyses of adult ARDS cohorts, and while initial studies are in progress (NCT03616704 and NCT03939260), an intervention targeting either parameter has yet to be proven efficacious.

To date, there has been no randomized trial to determine the appropriate application of any type of protective ventilation in pediatric ARDS, and observational studies offer conflicting results (6-10). Ventilator management in children is often extrapolated from adults, with uncertain applicability (10). Pediatric ARDS has a distinct epidemiology, with different inciting etiologies and predictors of outcome $(11,12)$, relative to adults, necessitating specific investigations in children. Overall, even less evidence is available for children regarding the utility of either MP or $\triangle \mathrm{P}$ as metrics of VILI or as modifiable ventilator parameters. 
To investigate how minimizing either MP or $\Delta \mathrm{P}$ would affect ventilator settings and gas exchange in ARDS, we employed a high-fidelity computational simulator matched to individual patient data from two separate cohorts, pediatric and adult. High-fidelity simulation holds the potential to develop, test, and directly compare ventilation strategies prior to exposing vulnerable patients to potentially damaging interventions (13). Global optimization algorithms, implemented on high-performance computing clusters, were used to evaluate more than 26.7 million different changes to the baseline ventilator settings to identify those that minimized $\Delta \mathrm{P}$, MP, and a modified formulation of MP (MMP) based on concerns (14) regarding the direct, positive, linear effect of positive end-expiratory pressure (PEEP) on MP in the original MP equation. Changes to ventilator settings were constrained within specified limits, and maximally protective settings optimizing $\triangle \mathrm{P}, \mathrm{MP}$, and MMP were calculated for two different scenarios (a) allowing, within safe limits, some deterioration in gas exchange from baseline, and (b) without allowing any deterioration in gas exchange. The primary aim of this study was to assess the scope for achieving more protective ventilation by separately minimizing $\triangle \mathrm{P}, \mathrm{MP}$, or MMP. A secondary goal of the study was to investigate to what extent protective ventilation strategies identified for the pediatric cohort were consistent with those computed for the adult cohort.

\section{MATERIALS AND METHODS}

\section{Patient Selection}

Pediatric cohort: Patients were selected from an ongoing (2011 onwards) prospective cohort (15) of intubated children meeting Berlin ARDS criteria from the Children's Hospital of Philadelphia (CHOP). The study was reviewed by the CHOP Institutional Review Board, and requirement for informed consent waived. Seventy-seven subjects between 1 month and 18 years 
of age (Mean: $3.1 \pm 3.3$ years, $23 \%$ severe, $44 \%$ moderate and 33\% mild ARDS), ventilated via cuffed endotracheal tube during neuromuscular blockade, were selected. Subjects were selected based on the initial development and validation of the pediatric algorithm. An initial development cohort of children with identically sized endotracheal tubes $(5.0 \mathrm{~mm}$ internal diameter) under neuromuscular blockade, and two subsequent test cohorts of infants under 2 years of age and children with $\mathrm{V}_{\mathrm{T}}>10 \mathrm{~mL} / \mathrm{kg}$. Arterial blood gases $(\mathrm{ABG})$ and ventilator changes during the first 72 hours of ARDS were recorded. All subjects were ventilated with decelerating flow in either pressure control or pressure-regulated volume control. Peak inspiratory pressure (PIP), PEEP, and exhaled $\mathrm{V}_{\mathrm{T}}$ were collected at the ventilator for patients with $\mathrm{V}_{\mathrm{T}} \geq 100 \mathrm{~mL}$ using integrated software provided by the manufacturer (Dräger, Inc., Lübeck, Germany), and using a sensor proximate to the endotracheal tube for $\mathrm{V}_{\mathrm{T}}<100 \mathrm{~mL}$.

Adult cohort: Data were extracted from 100 adult ARDS patients randomly selected (14\% severe, $66 \%$ moderate, $20 \%$ mild) from the low $\mathrm{V}_{\mathrm{T}}$ arm of the ARMA trial (16). Data were provided in a de-identified state by the Biologic Specimen and Data Repository Information Coordinating Centre the National Heart, Lung and Blood Institute, and informed consent was not required. All patients received mechanical ventilation in assist-control ventilation mode, and we used the earliest available post-randomization data.

\section{Simulator Calibration to Patient Data}

Analyses were carried out using a simulator that includes representations of multiple interacting organ systems, incorporates a high level of physiological detail, and has been extensively validated in several previous studies of adult $(17,18)$ and pediatric ARDS (13) (Supplemental File, section S1-S2). The simulator was matched to individual patient data 
(ventilator parameters and ABGs at single time points) using advanced global optimization algorithms, (Supplemental File, section S3). The optimal parameterisation of the simulator for each patient was used in all subsequent analyses (in the case of multiple parameterisations returning similar fits, robustness of the results was checked on the best 20).

\section{Maximally Protective Ventilation as a Constrained Optimization Problem}

After matching the model to each individual patient, the potential for achieving maximally lung-protective (but acceptably effective) ventilation was investigated by formulating and solving different optimization problems. We used advanced global optimization algorithms implemented on high-performance computing clusters to exhaustively search through more than 26.7 million different changes (approximately 150,000 per patient) to the reported ventilator settings - namely $\mathrm{V}_{\mathrm{T}}$, respiratory rate $(\mathrm{RR}), \mathrm{F}_{\mathrm{I}} \mathrm{O}_{2}, \mathrm{PEEP}$ and duty cycle (DC, inspiratory-to-total time ratio) to identify which settings produced minimum values of the following quantities:

- $\quad \Delta \mathrm{P}$ (difference between $\mathrm{P}_{\text {plat }}$ ) and PEEP), with $\mathrm{P}_{\text {plat }}$ is calculated directly from the simulator.

- Mechanical Power, defined as (1):

$$
M P=0.098 \times R R \times\left\{V_{T}^{2} \times\left[0.5 \times E L_{r s}+R R \times \frac{(1+I: E)}{60 \times I: E} \times R_{a w}\right]+V_{T} \times P E E P\right\}
$$

where $\mathrm{EL}_{\mathrm{rs}}$ is respiratory system elastance, $\mathrm{I}: \mathrm{E}$ is inspiratory-to-expiratory ratio, and $\mathrm{R}_{\mathrm{aw}}$ is the airway resistance. Note that, as shown in [1], the MP equation can also be simplified to:

$$
M P=0.098 \times R R \times V_{T} \times(P I P-0.5 \times \Delta P)
$$

- A modified version of MP, given by:

$$
M M P=0.098 \times R R \times V_{T}^{2} \times\left[0.5 \times E L_{r s}+R R \times \frac{(1+I: E)}{60 \times I: E} \times R_{a w}\right]
$$

which removes the direct linear, positive dependence between MP and PEEP (14). 
To ensure the relevance of these optimization problems to clinical practice, it is necessary to constrain the search for maximally protective settings to include only those that do not compromise oxygenation and ventilation. We did this by defining upper and lower limits for the ventilation settings themselves, and by defining allowable limits for the values of PIP, $\mathrm{P}_{\mathrm{a}} \mathrm{O}_{2}$ and $\mathrm{P}_{\mathrm{a}} \mathrm{CO}_{2}$ produced by the settings (Table 1 ). Ventilation settings that minimized $\triangle \mathrm{P}, \mathrm{MP}$ and MMP while keeping values of PIP, $\mathrm{P}_{\mathrm{a}} \mathrm{O}_{2}$ and $\mathrm{P}_{\mathrm{a}} \mathrm{CO}_{2}$ within their specified limits were computed for each patient (Approach 1). In the pediatric cohort, these limits were based on those used in the ARDSNetwork trial, adapted to match pediatric conventions $(6,8,10,19)$. As the pediatric cohort was developed using decelerating flow, as is most common in pediatrics (20), PIP was used as a constraint, rather than $\mathrm{P}_{\text {plat }}$. When data indicated that a patient's initial ventilator state did not comply with one or more of the specified safety limits, changes to the settings were only made if they led to an improvement in the relevant parameters (e.g., reducing $\mathrm{P}_{\mathrm{a}} \mathrm{CO}_{2}$ or PIP).

As an alternative strategy, we also investigated whether changes to ventilator settings could be found that minimized $\Delta \mathrm{P}, \mathrm{MP}$ and MMP without resulting in any deterioration in $\mathrm{P}_{\mathrm{a}} \mathrm{O}_{2}$ and $\mathrm{P}_{\mathrm{a}} \mathrm{CO}_{2}$ from baseline values (Approach 2). An upper limit of $35 \mathrm{cmH}_{2} \mathrm{O}$ was applied for PIP in the pediatric cohort. Due to relatively higher baseline PIP in adults, the upper limit was set to the corresponding baseline values for these patients (Supplemental File, section S6).

\section{Statistical Analysis}

Data are presented as mean \pm standard deviation $(\mathrm{SD})$, or shown graphically using median, interquartile and total ranges. To avoid violation of underlying distribution assumptions, variables were compared using the signed-rank test. A two-sided p-value of $<0.05$ was considered significant. 


\section{RESULTS}

\section{The Simulator Accurately Represents Individual Patient Data}

The ability of the simulator to reproduce patient data was verified by comparing its responses $\left(\mathrm{P}_{\mathrm{a}} \mathrm{O}_{2}\right.$ and $\left.\mathrm{P}_{\mathrm{a}} \mathrm{CO}_{2}\right)$ against data on the responses of patients from both cohorts. After calibration (Supplemental File, section S3), each patient in the cohort was simulated for 30 minutes (or until reaching steady-state) under volume controlled mechanical ventilation with constant flow in the supine position. Figure 1-(A) to 1-(C) compares the outputs of the simulator with the original data, expressed as median, interquartile range and actual range for the entire cohort. Figure 1-(D) to 1-(F) shows the Bland-Altman plots for data points versus simulator output values. These results confirm the capability of the simulator to accurately replicate multiple output values of the patients included in both cohorts across a range of different ventilator settings.

\section{Reductions in $\triangle P$, MP, and MMP were Achieved in Both Cohorts}

When ABGs were allowed to vary within pre-specified ranges (Table 1), average maximum reductions in $\Delta \mathrm{P}$ of $3.0 \pm 2.2 \mathrm{cmH}_{2} \mathrm{O}(23 \%)$ compared to baseline values in the pediatric cohort and $3.2 \pm 2.1 \mathrm{cmH}_{2} \mathrm{O}(23 \%)$ in the adult cohort were achievable (Figure 2). Reductions in $\Delta \mathrm{P}$ of over $1 \mathrm{cmH}_{2} \mathrm{O}$ were achieved in $95 \%$ of pediatric and $82 \%$ of adult patients. The corresponding reductions when targeting MP were $3.3 \pm 2.6 \mathrm{~J} \cdot \mathrm{min}^{-1}(44 \%)$ in the pediatric cohort (87\% of whom had MP reduced by over $20 \%)$ and $21.0 \pm 5.4 \mathrm{~J} \cdot \mathrm{min}^{-1}(66 \%)$ in the adult cohort, with all patients reducing MP by over $20 \%$. When targeting MMP, reductions were $3.7 \pm$ 2.3 J.min ${ }^{-1}(61 \%)$ in the pediatric cohort and $15.2 \pm 4.9 \mathrm{~J} \cdot \mathrm{min}^{-1}(67 \%)$ in the adult cohort 
(reductions of over $20 \%$ in $95 \%$ and $99 \%$ of the pediatric and adult cohorts, respectively).

Reductions were statistically significant in all groups (signed-rank test $\mathrm{p}<0.05$ ). In all the above cases, more protective ventilation was achieved with no significant deterioration in patient oxygenation $\left(\mathrm{P}_{\mathrm{a}} \mathrm{O}_{2}\right)$, although $\mathrm{P}_{\mathrm{a}} \mathrm{CO}_{2}$ did consistently increased towards the upper limits (Supplemental File, Figure S5). In both cohorts, settings that minimized $\Delta \mathrm{P}$ also reduced MP and MMP, whereas settings that minimized MP and MMP increased $\Delta \mathrm{P}$ (largely due to the resulting increases in $\mathrm{V}_{\mathrm{T}}$ (see below).

When the optimizations were constrained to allow no deterioration in gas exchange (i.e. only changes that maintained, or improved, $\mathrm{PaO}_{2}$ and $\mathrm{PaCO}_{2}$ with respect to baseline values), reductions were achievable in $\Delta \mathrm{P}$ of $1.6 \pm 1.4 \mathrm{cmH}_{2} \mathrm{O}(12 \%)$ and $0.4 \pm 1.0 \mathrm{cmH}_{2} \mathrm{O}(2 \%)$ were achievable compared to baseline values in the pediatric and adult cohorts, respectively (Figure 3). Reductions of $\Delta \mathrm{P}$ of over $1 \mathrm{cmH}_{2} \mathrm{O}$ were achieved in $58 \%$ of pediatric and $16 \%$ of adult subjects. Corresponding reductions when targeting MP were $1.7 \pm 1.4 \mathrm{~J} \cdot \mathrm{min}^{-1}(24 \%)$ in the pediatric cohort and $14.4 \pm 4.9 \mathrm{~J} \cdot \mathrm{min}^{-1}(46 \%)$ in the adult cohort, with $57 \%$ of pediatric and $98 \%$ of adult patients having MP reduced by over $20 \%$. When targeting MMP, the reductions achievable were $2.5 \pm 1.5 \mathrm{~J} \cdot \mathrm{min}^{-1}(44 \%)$ in the pediatric cohort (90\% of whom had reductions of more than 20\%) and $10.3 \pm 4.4 \mathrm{~J}_{\mathrm{min}} \mathrm{m}^{-1}(46 \%)$ in the adult cohort (97\% achieving reductions of more than 20\%). Reductions were significant in all cases (signed-rank test $\mathrm{p}<0.05$ ).

\section{Minimum values of $\Delta \mathbf{P}$ and MP are Achieved by Distinct Ventilation Strategies}

Minimum values of MP in both adult and pediatric cohorts were produced by increased $\mathrm{V}_{\mathrm{T}}\left(\right.$ Pediatric: $1.4 \pm 1.8 \mathrm{~mL} \cdot \mathrm{kg}^{-1}(+19 \%)$, Adult: $\left.1.9 \pm 1.1 \mathrm{~mL} \cdot \mathrm{kg}^{-1}(+34 \%)\right)$, decreased RR (Pediatric: $-8.6 \pm 5.1 \mathrm{bpm}(-34 \%)$, Adult: $-15.6 \pm 5.0 \mathrm{bpm}(-56 \%)$ ), DC at or close to its specified 
upper limit of 0.6, and PEEP at or close to its specified lower limit of $5 \mathrm{cmH}_{2} \mathrm{O}$ (Figure 4). $\mathrm{F}_{\mathrm{I}} \mathrm{O}_{2}$ increased in both pediatric and adult cohorts (Pediatric: $+39 \%$, Adult: $+26 \%$ ).

Similar changes in $\mathrm{V}_{\mathrm{T}}$, RR and DC were observed in both cohorts when targeting the MMP. As expected, minimizing MMP rather than MP resulted in higher values of PEEP in both pediatric and adult cohorts (Pediatric: $2.9 \pm 4.6 \mathrm{cmH}_{2} \mathrm{O}(+39 \%)$, Adult: $3.5 \pm 5.0 \mathrm{cmH}_{2} \mathrm{O}$ $(+52 \%))$ along with lower values of $\mathrm{F}_{\mathrm{I}} \mathrm{O}_{2}$ in pediatric patients $(-21 \%)$.

In the pediatric cohort, minimum $\Delta \mathrm{P}$ was achieved by reducing $\mathrm{V}_{\mathrm{T}}\left(1.3 \pm 1.6 \mathrm{~mL} \cdot \mathrm{kg}^{-1}(-\right.$ $15 \%))$ while increasing RR and PEEP $\left(2.3 \pm 8.2 \mathrm{bpm}(+11 \%)\right.$ and $2.4 \pm 4.5 \mathrm{cmH}_{2} \mathrm{O}(+34 \%)$ respectively). In the adult cohort, no reductions in $\mathrm{V}_{\mathrm{T}}$ were possible, but $\Delta \mathrm{P}$ could still be reduced by increasing PEEP $\left(2.2 \pm 3.5 \mathrm{cmH}_{2} \mathrm{O}(+32 \%)\right)$. No changes in DC were observed in either cohort when targeting $\Delta \mathrm{P}$. Patterns of changes in ventilator settings were consistent in most cases between Approach 1 (allowing some deterioration in blood gas values; Figure 4) and Approach 2 (allowing no deterioration in blood gas values; Supplemental File, Figure S7), although when minimizing $\Delta \mathrm{P}$ in pediatric patients, Approach 2 produced higher values of $\mathrm{F}_{\mathrm{I}} \mathrm{O}_{2}$ than Approach 1, in order to satisfy the requirement for no deterioration in oxygenation.

\section{DISCUSSION}

Our results provide several new insights into the types of ventilation strategies that are likely to promote lung protective ventilation in ARDS patients. A high degree of consistency was observed in settings that minimized $\triangle \mathrm{P}, \mathrm{MP}$, and MMP across the diverse patient cohorts in both datasets, providing grounds for optimism that strategies for maximally protective ventilation could be developed that would be widely applicable in ARDS. 
Perhaps the most counterintuitive result is that maximum reductions in MP and MMP are consistently achieved by increasing $\mathrm{V}_{\mathrm{T}}$, (see Figures $\mathrm{S} 7$ and $\mathrm{S} 8$ ), since from both the standard (Equation 1) and modified (Equation 3) formula for MP it seems obvious that lowering $\mathrm{V}_{\mathrm{T}}$ should lower MP. Crucially, however, this ignores the impact of incorporating constraints on allowable deterioration in patient gas exchange, which would always exist in treatment strategies implemented at the bedside. These constraints, combined with the complexity of making simultaneous adjustments to multiple ventilator settings, add a host of other trade-offs that render the optimal combination of ventilator settings almost impossible to predict based on clinical intuition alone. Our results point to a complex interplay between ventilator parameters which would support the development of a closed-loop system that can incorporate direct patient inputs, thereby providing individualized safe and effective mechanical ventilation.

In the pediatric cohort, minimum values of $\Delta \mathrm{P}$ were achieved by reducing $\mathrm{V}_{\mathrm{T}}$ and increasing RR and PEEP. This strategy has much in common with the ARMA trial protocol, which also lowered $\mathrm{V}_{\mathrm{T}}$ while increasing RR. Some have postulated that this combination led to increased intrinsic (and hence total) PEEP (21), which may have contributed to the mortality benefit in this trial. However, it should be noted that subsequent trials of higher versus lower PEEP have not demonstrated a mortality benefit in heterogeneous ARDS populations (22-25). Since the selected patients in the adult cohort were from the low $\mathrm{V}_{\mathrm{T}}$ arm of the trial, no further reductions in $\mathrm{V}_{\mathrm{T}}$ were possible without violating imposed constraints on gas exchange. However, $\Delta \mathrm{P}$ could still be significantly reduced in this cohort by moderately increasing PEEP.

Our findings provide novel insights into the challenges of using either $\Delta \mathrm{P}$ or MP to develop protective ventilatory strategies. In our models, targeting reductions in $\Delta \mathrm{P}$ led to increased RR and increased PEEP. While a strong association between higher $\Delta \mathrm{P}$ and mortality 
has been demonstrated $(4,26,27), \Delta \mathrm{P}$ was not a therapeutic target in these patients, and causality remains elusive. There is data suggesting that increasing RR (28) and PEEP (29) beyond safe thresholds can be deleterious in injured lungs. Furthermore, in the Alveolar Recruitment for ARDS Trial (ART) clinical trial (25), a ventilatory strategy that decreased $\Delta \mathrm{P}$ resulted in increased mortality. The usefulness of targeting $\Delta \mathrm{P}$ directly thus remains to be demonstrated.

These concerns also apply to strategies that target reductions in MP. While MP represents a more complete attempt to describe the contributions of multiple parameters to VILI by invoking their "energy cost," the relative contributions of the different parameters (i.e., their relatively equal "weightings" in the formula) remains the subject of debate. An example is the controversy around how PEEP contributes to MP (14). Our results show that different formulations of the MP equation lead to different optimal strategies; specifically, higher PEEP when optimizing MMP. Our finding that strategies that minimize MP and MMP increase $\mathrm{V}_{\mathrm{T}}$ highlights the challenges of targeting one specific parameter in designing protective ventilatory strategies. This is particularly important given the findings from recent pre-clinical animal studies that, for the same MP, strategies employing higher rather than lower $\mathrm{V}_{\mathrm{T}}$ had increased injury $(30,31)$. All these findings highlight the need for prospective validation of ventilator strategies that target reduced MP. Of importance, computational modelling of the impact of targeting these parameters (or combinations of different VILI indices) may identify promising non-intuitive combinations of ventilator settings for clinical testing, and also allow more effective stratification of patient populations by revealing differences in the effects of ventilation strategies across heterogeneous patient populations. We note that current ventilators do not routinely calculate and display MP, and that the ability to do so would improve tracking of this parameter for both clinical and research purposes. 
Our study has a number of limitations. The pediatric dataset was derived from a single institution, and while the severity of ARDS and outcomes were similar to other cohorts, generalizability cannot be assumed. To minimize confounding, the model was configured to represent patients who are fully sedated and/or paralyzed; therefore, autonomic reflex modules were not utilized. In both cohorts, for each patient the model was "trained" on a single dataset (i.e., ventilator settings and blood gasses recorded at a single time point) and model calculations regarding the effect of other ventilator settings on, for example, lung compliance, are predictions that assume an unchanged patient physiological state. The model also does not include the effect of inflammatory mediators, which are difficult to quantify and to isolate in clinical settings. As the model is computational in nature, it does not provide any direct physiologic, histological or biological evidence of the effects of the proposed ventilation strategies on VILI, and further animal and human studies should be performed to provide conclusive evidence of their effectiveness in achieving more protective ventilation. The model was developed to focus on ventilator settings affecting VILI; thus, we chose to set constraints on $\mathrm{P}_{\mathrm{a}} \mathrm{CO}_{2}$, rather than $\mathrm{pH}$, which is often modified by entirely non-ventilator interventions, such as volume resuscitation or exogenous bicarbonate. Finally, models were based on ventilator settings and ABGs at single timepoints, and not on prospective data collection after planned ventilator changes. Such a study design would provide a more granular data regarding an individual patient's response to specific ventilator adjustments.

However, our study also has several unique strengths. Over 26.7 million distinct combinations of ventilator settings were implemented and evaluated on two separate cohorts of patients with ARDS. It is difficult to imagine such a comprehensive exploration of different ventilation strategies ever being possible via animal or clinical trials. The study also allows a 
direct comparison of the effects of protective ventilation strategies in adult and pediatric ARDS patients. Our results clearly demonstrate the utility of pilot studies using high-fidelity simulation to assess novel interventions targeting MP or $\triangle \mathrm{P}$ (or any other VILI indicator), and hence to inform the design of more targeted and effective clinical trials on actual patients.

\section{CONCLUSIONS}

We identified novel ventilatory strategies that our model predicts will $\triangle \mathrm{P}, \mathrm{MP}$, and MMP in datasets from adults and children with ARDS. The identified strategies were consistent within each patient group, and were similar in both adults and children, suggesting that protective ventilatory strategies derived from studies in adults may have utility in children with ARDS. Our model predicts that attempts to minimize MP could result in the use of higher $\mathrm{V}_{\mathrm{T}}$. Since this contradicts the current consensus on using lower $\mathrm{V}_{\mathrm{T}}$ it raises questions regarding the use of MP as a direct target to minimize VILI, at least as currently formulated. Overall, our findings demonstrate the limitations of ventilatory strategies that target either $\Delta \mathrm{P}$ or $\mathrm{MP}$, highlighting the need to continue to refine these targets, and for ultimate validation of these strategies in clinical trials. 


\section{REFERENCES}

1. Gattinoni L, Tonetti T, Cressoni M, et al. Ventilator-related causes of lung injury: the mechanical power. Intensive Care Med 2016;42(10):1567-1575.

2. Vasques F, Duscio E, Pasticci I, et al. Is the mechanical power the final word on ventilator-induced lung injury?-we are not sure. Ann Transl Med 2018;6(19):395.

3. Serpa Neto A, Deliberato RO, Johnson AEW, et al. Mechanical power of ventilation is associated with mortality in critically ill patients: an analysis of patients in two observational cohorts. Intensive Care Med 2018;44(11):1914-1922.

4. Amato MB, Meade MO, Slutsky AS, et al. Driving pressure and survival in the acute respiratory distress syndrome. N Engl J Med 2015;372(8):747-755.

5. Das A, Camporota L, Hardman JG, et al. What links ventilator driving pressure with survival in the acute respiratory distress syndrome? A computational study. Respir Res 2019;20(1):29.

6. Erickson S, Schibler A, Numa A, et al. Acute lung injury in pediatric intensive care in Australia and New Zealand: a prospective, multicenter, observational study. Pediatr Crit Care Med 2007;8(4):317-323.

7. Albuali WH, Singh RN, Fraser DD, et al. Have changes in ventilation practice improved outcome in children with acute lung injury? Pediatr Crit Care Med 2007;8(4):324-330.

8. Khemani RG, Conti D, Alonzo TA, et al. Effect of tidal volume in children with acute hypoxemic respiratory failure. Intensive Care Med 2009;35(8):1428-1437.

9. de Jager P, Burgerhof JG, van Heerde M, et al. Tidal volume and mortality in mechanically ventilated children: a systematic review and meta-analysis of observational studies*. Crit Care Med 2014;42(12):2461-2472. 
10. Yehya N, Thomas NJ. Disassociating Lung Mechanics and Oxygenation in Pediatric Acute Respiratory Distress Syndrome. Crit Care Med 2017;45(7):1232-1239.

11. Yehya N, Keim G, Thomas NJ. Subtypes of pediatric acute respiratory distress syndrome have different predictors of mortality. Intensive Care Med 2018;44(8):1230-1239.

12. Khemani RG, Smith L, Lopez-Fernandez YM, et al. Paediatric acute respiratory distress syndrome incidence and epidemiology (PARDIE): an international, observational study. The Lancet Respiratory medicine 2019;7(2):115-128.

13. Saffaran S, Das A, Hardman JG, et al. High-fidelity computational simulation to refine strategies for lung-protective ventilation in paediatric acute respiratory distress syndrome. Intensive Care Med 2019;45(7):1055-1057.

14. Huhle R, Serpa Neto A, Schultz MJ, et al. Is mechanical power the final word on ventilator-induced lung injury?-no. Ann Transl Med 2018;6(19):394.

15. Yehya N, Thomas NJ, Khemani RG. Risk Stratification Using Oxygenation in the First 24 Hours of Pediatric Acute Respiratory Distress Syndrome. Crit Care Med 2018;46(4):619-624. 16. Acute Respiratory Distress Syndrome N, Brower RG, Matthay MA, et al. Ventilation with lower tidal volumes as compared with traditional tidal volumes for acute lung injury and the acute respiratory distress syndrome. N Engl J Med 2000;342(18):1301-1308.

17. Das A, Cole O, Chikhani M, et al. Evaluation of lung recruitment maneuvers in acute respiratory distress syndrome using computer simulation. Crit Care 2015;19:8.

18. Chikhani M, Das A, Haque M, et al. High PEEP in acute respiratory distress syndrome: quantitative evaluation between improved arterial oxygenation and decreased oxygen delivery. British journal of anaesthesia 2016;117(5):650-658. 
19. Khemani RG, Markovitz BP, Curley MAQ. Characteristics of children intubated and mechanically ventilated in 16 PICUs. Chest 2009;136(3):765-771.

20. Santschi M, Jouvet P, Leclerc F, et al. Acute lung injury in children: therapeutic practice and feasibility of international clinical trials. Pediatr Crit Care Med 2010;11(6):681-689.

21. de Durante G, del Turco M, Rustichini L, et al. ARDSNet lower tidal volume ventilatory strategy may generate intrinsic positive end-expiratory pressure in patients with acute respiratory distress syndrome. Am J Respir Crit Care Med 2002;165(9):1271-1274.

22. Brower RG, Lanken PN, MacIntyre N, et al. Higher versus lower positive end-expiratory pressures in patients with the acute respiratory distress syndrome. N Engl J Med 2004;351(4):327-336.

23. Mercat A, Richard JC, Vielle B, et al. Positive end-expiratory pressure setting in adults with acute lung injury and acute respiratory distress syndrome: a randomized controlled trial. JAMA 2008;299(6):646-655.

24. Meade MO, Cook DJ, Guyatt GH, et al. Ventilation strategy using low tidal volumes, recruitment maneuvers, and high positive end-expiratory pressure for acute lung injury and acute respiratory distress syndrome: a randomized controlled trial. JAMA 2008;299(6):637-645.

25. Writing Group for the Alveolar Recruitment for Acute Respiratory Distress Syndrome Trial I, Cavalcanti AB, Suzumura EA, et al. Effect of Lung Recruitment and Titrated Positive End-Expiratory Pressure (PEEP) vs Low PEEP on Mortality in Patients With Acute Respiratory Distress Syndrome: A Randomized Clinical Trial. JAMA 2017;318(14):1335-1345.

26. Bellani G, Laffey JG, Pham T, et al. Epidemiology, Patterns of Care, and Mortality for Patients With Acute Respiratory Distress Syndrome in Intensive Care Units in 50 Countries. JAMA 2016;315(8):788-800. 
27. Laffey JG, Bellani G, Pham T, et al. Potentially modifiable factors contributing to outcome from acute respiratory distress syndrome: the LUNG SAFE study. Intensive Care Med 2016;42(12):1865-1876.

28. Retamal J, Borges JB, Bruhn A, et al. High respiratory rate is associated with early reduction of lung edema clearance in an experimental model of ARDS. Acta Anaesthesiol Scand 2016;60(1):79-92.

29. Retamal J, Bugedo G, Larsson A, et al. High PEEP levels are associated with overdistension and tidal recruitment/derecruitment in ARDS patients. Acta Anaesthesiol Scand 2015;59(9):1161-1169.

30. Moraes L, Silva PL, Thompson A, et al. Impact of Different Tidal Volume Levels at Low Mechanical Power on Ventilator-Induced Lung Injury in Rats. Front Physiol 2018;9:318.

31. Santos RS, Maia LA, Oliveira MV, et al. Biologic Impact of Mechanical Power at High and Low Tidal Volumes in Experimental Mild Acute Respiratory Distress Syndrome. Anesthesiology 2018;128(6):1193-1206. 


\section{Figure Legends:}

Figure 1: Panels (A) to (C) compare the simulator outputs versus patient data expressed as median, interquartile ranges and actual ranges. Panels (D) to (F) show the Bland-Altman plots for simulator outputs and data. $\mathrm{R}$ represents the correlation coefficient of the data and the simulated values.

Figure 2: Approach 1 - Change in driving pressure, mechanical power and modified mechanical power when minimizing different targets (i.e. $\triangle \mathrm{P}, \mathrm{MP}$ and MMP) and allowing some deterioration in patient gas exchange. Panels (A) to (C) show results for the pediatric cohort and (D) to (F) for the adult cohort. Box plots demonstrate data as median, interquartile range and actual. Numbers on the whiskers are mean \pm SD. $\Delta \mathrm{P}$ : Driving Pressure; MP: Mechanical Power; MMP: Modified Mechanical Power. The corresponding changes in tidal volume, respiratory rate, duty cycle, $\mathrm{F}_{\mathrm{I}} \mathrm{O}_{2}$ and PEEP are shown in Fig. S7 and S8.

Figure 3: Approach 2 - Change in driving pressure, mechanical power and modified mechanical power when minimizing different targets (i.e. $\triangle \mathrm{P}, \mathrm{MP}$ and $\mathrm{MMP}$ ) and allowing no deterioration in gas-exchange. Panels (A) to (C) show results for the pediatric cohort and (D) to (F) for the adult cohort. Box plots demonstrate data as median, interquartile range and actual. Numbers on the whiskers are mean \pm SD. $\triangle \mathrm{P}$ : Driving Pressure; MP: Mechanical Power; MMP: Modified Mechanical Power. The corresponding changes in tidal volume, respiratory rate, duty cycle, $\mathrm{F}_{\mathrm{I}} \mathrm{O}_{2}$ and PEEP are shown in Fig. S7 and S8. 